\title{
The Refinement of Microcalcification Cluster Assessment By Joint Analysis of MLO and CC views
}

\author{
Márta Altrichter and Gábor Horváth \\ Department of Measurement and Information Systems, \\ Budapest University of Technology and Economics, \\ P.O.Box 91, H-1521 Budapest, Hungary \\ grimma@sch.bme.hu, horvath@mit.bme.hu
}

\begin{abstract}
Most of the CAD Systems for Mammograms are composed of algorithms analysing the four X-ray images individually. It is a general experience, that algorithms in search of microcalcification clusters can obtain high sensitivity only if specificity is low. To overcome efficiency problem this paper proposes a simple algorithm to combine information of the two views (MLO/CC) of the breast. The procedure is based upon the experiences of radiologists: masses and calcifications should emerge on both views, so if no matching is found, the given object is a false positive hit. A positioning system is evolved to find corresponding regions on the two images. Calcification clusters obtained in individual images are matched in "2.5-D" provided by the positioning system. The credibility value of the hit is reassessed by the matching. The proposed approach can significantly reduce the number of false positive hits in calcification.
\end{abstract}

\section{Introduction}

There are several algorithms searching for microcalcification clusters on individual X-ray images [1], [2]. The main feature of these algorithms is that the positive cases are found with large probability - sensitivity is about $90-95 \%$ but the number of false positive hits per picture is too high - 2-4 FP/image, specificity $0-5 \%$.

A method is needed to decrease the number of false positive hits, which will not or will barely decrease the number of true positive ones. This paper presents a relatively simple new way of this. The method sets off from the fact that the images of calcifications and masses have to appear on both views (MLO and CC). To be more precise they must be on positions of the two views that correspond to each other. In practice a 3-D reconstruction of the breast would be needed. But the full 3-D reconstruction is impossible, because only two views of the breast are available, and because these two views are the 2-D projections of the differently compressed breast. Therefore instead of a full 3-D reconstruction we suggest a relatively simple procedure which we call " 2.5 - $\mathrm{D}$ " correspondence.

As the two main pathological abnormalities have different distinguishing features from normal tissue, their joint analysis slightly differs. During matching 
of masses the positioning system can restrict the examined picture to a region corresponding on the other view, and search within it for a mass with similar texture characteristics. On the other hand calcificated tissue and normal tissues are rather similar in texture, therefore their matching only uses the positioning system.

In this paper we focused on describing how the "2.5-D" positioning system can be built, and how it can be used to refine the assessment of a micocalcification cluster found previously by a microcalcification searching algorithm.

\section{2 "2.5-D" Positioning System}

The breast has three main control points: the pectoral muscle, the nipple and the boundary of the breast. These landmarks segment the breast to its anatomical regions.

The Cranio-Caudal and Medio-Lateral-Oblique views are two-dimensional projections of the three dimensional object. As the breast in $\mathrm{CC}$ view is exposed to x-ray from a different angle than in MLO, it can be assumed that a stripe will correspond on the MLO to a region taken from the $\mathrm{CC}$ view (this assumtion works backward as well, thus an MLO region transfered to the CC view is a stripe). The reference system is to calculate the position of this stripe. The algorithm is founded on three simple hypotheses:

1. The pectoral muscle on a $\mathrm{CC}$ image is the vertical axis.

2. The position of the nipple can be estimated by laying a tangent on the breast border parallel with the pectoral muscle.

3 . The distance covered from the nipple perpendicular to the pectoral muscle on MLO approximately corresponds to the distance measured up on the horizontal axis from the nipple on CC.

The first step of the algorithm is to find the angle enclosed by the pectoral muscle and the horizontal axis on MLO views.

To find the angle the slightly modified method in [3] was used. First edge detection - special edge detection method: Edgeflow [4] - is made and the Region of Interest (ROI) is cut out. This ROI is the upper corner of the MLO and will contain the pectoral muscle (See Fig. 1(a).).

The second step: the iteration processing the lines in the ROI diverges from paper [3], as in this case the whole line of the pectoral muscle is not needed, just an approximation of the angle enclosed by the pectoral muscle and the horizontal axis. By cutting up the lines, and deleting improbable line segments the robustness of the algorithm is increased. (See Fig. 1.) The pseudo code is:

1. $n=0, B W_{0}=R O I, L_{n}=$ longest object on $B W_{0}$

2. $L_{n}$ is divided to parts with uniform length along the vertical axis

3. $L_{-} b a d_{n}=$ objects which enclose $<40^{\circ}$ or $>90^{\circ}$ with the horizontal axis

4. $L_{-} \operatorname{good}_{n}=L_{n}-L_{-} b a d_{n}, B W_{n+1}=B W_{n}-L \_b a d_{n}$

5. if $B W_{n+1}==B W_{n}$ iteration stops, the pectoral muscle is the object $L_{-} \operatorname{good}_{n}$, else $L_{n+1}=$ longest object on $B W_{n+1}, n=n+1$ and go to Step 2 . 


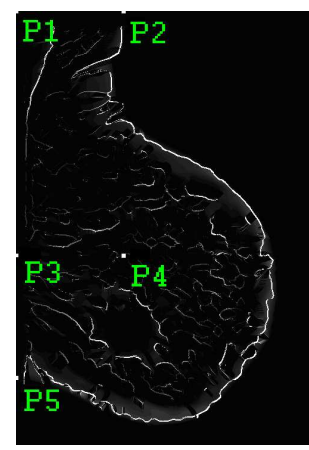

(a)

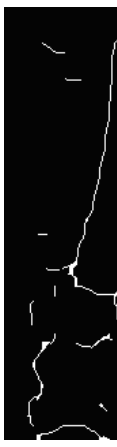

(b)

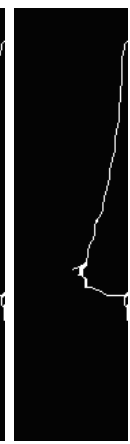

(c)

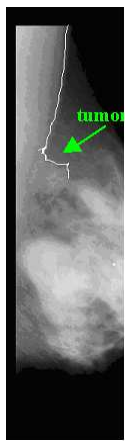

(d)

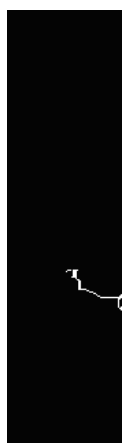

(e)

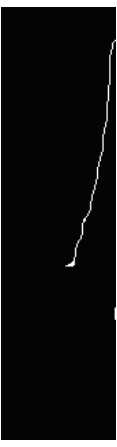

(f)

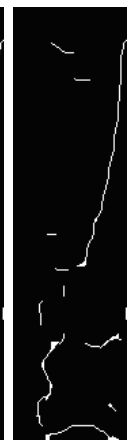

(g)

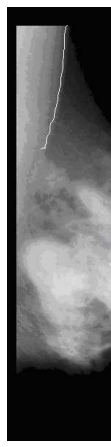

(h)

Fig. 1. (a) ROI selection (b) $B W_{0}$, (c) $L_{0}$, (d) $L_{0}+$ Picture, (e) $L \_b a d_{0}$, (f) $L_{-} g o o d_{1}$, (g) $B W_{1}$, (h) $L_{1}+$ Picture

After finding the angle a tangent with the same angle is laid on the breast border marking the nipple. The distances of the observed region from the tangent $-u$ and $v$ - are measured. The same distances are measured up on the perpendicular line to the tangent from the nipple of the other view. The two points and the angle of the pectoral muscle mark out the stripe needed for matching calcifications. (See Fig. 2.)

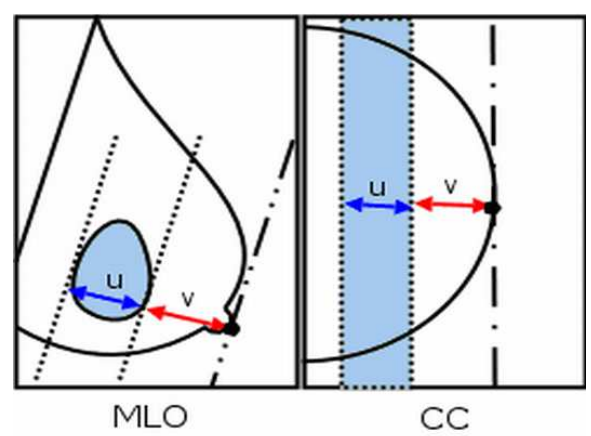

Fig. 2. The corresponding stripe on the $\mathrm{CC}$ of a selected region on the MLO

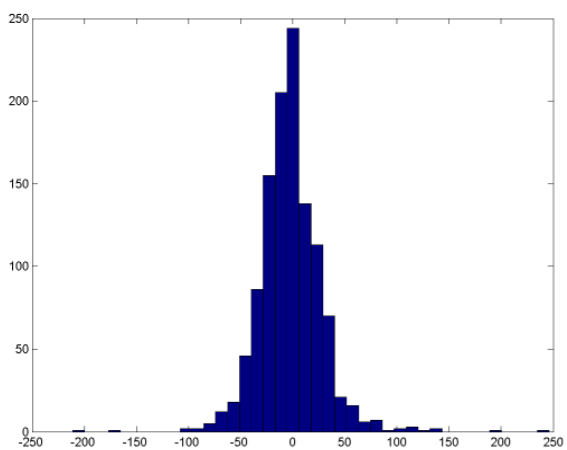

Fig. 3. Histogram of pixel errors, number of cases 1159

To test if the three basic hypotheses of the positioning system are correct a statistical analysis was made. 1159 cases with $400 \mu /$ pixel resolution $\left(600^{*} 400\right.$ pixels) from the DDSM database [5] were selected. These cases contained only one pathological growth on each views according to the radiologists' assessments. Thus the two marks on the two views can be assumed to be the 2-D projections of the same object. 
The pixel corresponding to the centroid of the growth on the MLO was determined, and the deviation of the result from the centroid of the growth on the $\mathrm{CC}$ was measured in pixe $\equiv$ e Fig. 4.). Fig. 3. shows the histogram of the deviations. There is some varlance caused by wrong pectoral muscle finding, wrong radiologist assessment or the flaw of the hypotheses (because of breast deformation) for a few cases, but generally the hypotheses look to be standing (mean is around zero). To compensate the effect of variance the width of the stripe is increased by $10 \%$.

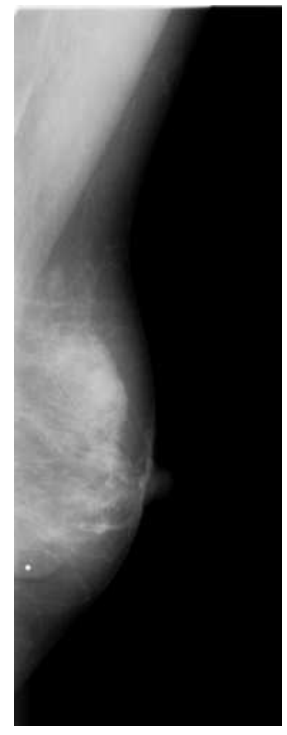

(a)

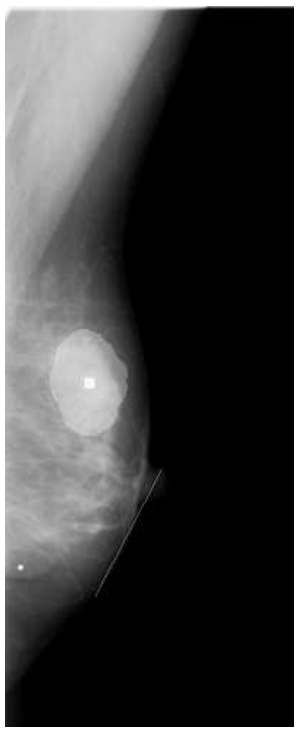

(b)

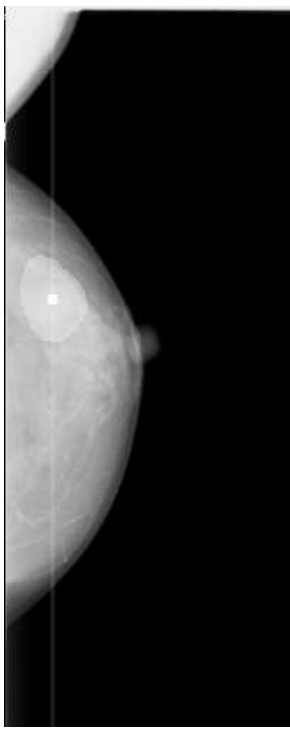

(c)

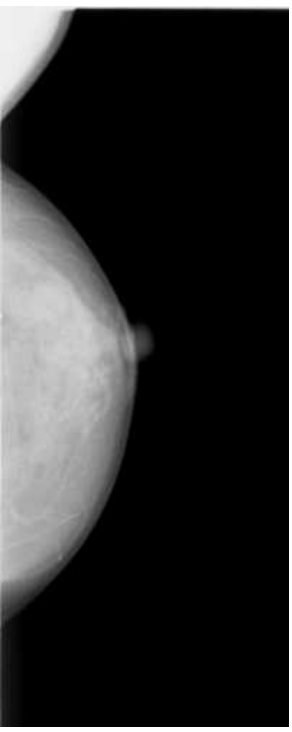

(d)

Fig. 4. (a) Original MLO (b) centroid of mass (marked by radiologist) is a white square (c) mass is marked by doctor + line corresponding to mass centroid on MLO (d) Original CC

To see if there is no inbuilt error from the pectoral muscle and nipple search, we have constructed a simple sensitivity test using the above mentioned 1159 cases and evaluation method. In the sensitivity test, a constant running from -50 to +40 was added to the pectoral muscle degree in each of the 1159 cases (90*1159 measures), the new nipple was calculated and the same deviations of pairing the centroids of radiologists' assessments were measured as in the statistical analysis (See Fig. 4.). Beside of these 90 measurement systems, an experiment on the microcalcification searching algorithm showed that the average cluster diameter is 52 pixe $\equiv$ the variance of the diameter is 25 pixel. On Fig. 5. we can see the percentage of the 1159 cases which are below the 27,52,77 pixel deviation plotted according to the constant change of the pectoral muscle angle. The figure shows two important facts: (a) there is no inbuilt constant 
error in the "2.5-D" positioning system, (b) there is quite big tolerance (around -10 to 10 degree) in finding the pectoral muscle angle.

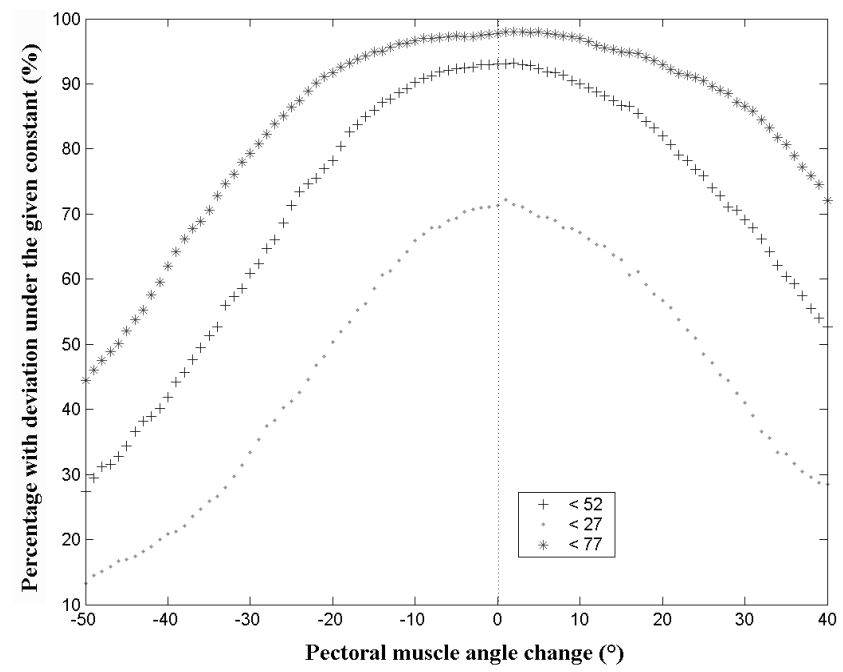

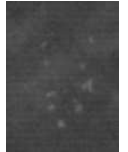

(a)

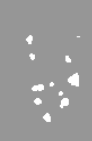

(b)

Fig. 6. (a) the original image part, (b) the calcification mask the searching algorithm provided

Fig. 5. The percentage of the 1159 cases which are below the constant $(27,52$ or 77$)$ ploted along the error in the pectoral muscle angle imbued by the experiment

\section{Microcalcification cluster matching and reassessment}

The microcalcification searching algorithm marks out suspicious regions, and provides a mask of microcacification pixels (See Fig. 6.). From the number of calcifications in the cluster, and the difference between the mean intensity of calcifications (marked by the mask) and the surrounding tissues a credibility value is assigned to each cluster: $P_{\text {calc }}$ (Range: 0-255, 255 - highly suspicious region).

This credibility is modified by the help of the area ratio of the stripe corresponding to it and of other calcification clusters found on the other view: $A_{\text {ratio }}$. The new credibility is: $\hat{P}_{\text {calc }}=P_{\text {calc }}-$ cons $_{1} *\left(1-A_{\text {ratio }}+\right.$ cons $\left._{2}\right)$. Fig. 8. illustrates why probability is decreased by subtracting an amount instead of simply multiplying with $A_{\text {ratio }}$. In this way if the microcalcification searching algorithm found the true positive cluster only on one view, but with high $P_{\text {calc }}$, the decreased $\hat{P}_{\text {calc }}$ is still thresholded to be a calcification. The constant cons $s_{2}$ is to ensure, that at high correspondence the suspicion is raised. 


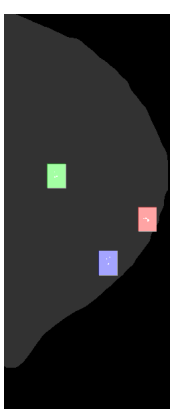

(a)

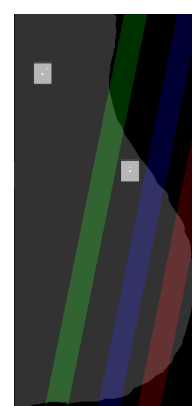

(b)

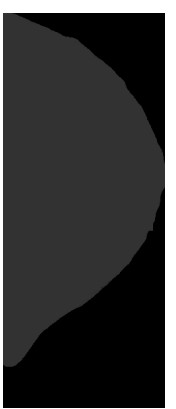

(c)

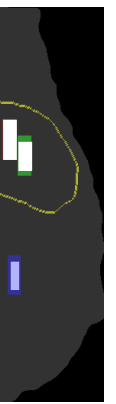

(a)

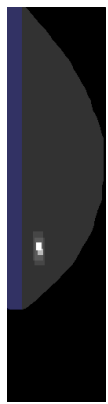

(b)

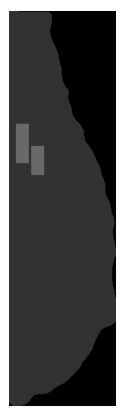

(c)

Fig. 7. A Normal Case is found because $P_{\text {calc }}$ of the $3 \mathrm{FP}$ mark is decreased below threshold for calcification

Fig. 8. True positive case is not lost although no matching. Yellow half-circe is the mark of the radiologist.

\section{Performance}

The calcification matching was analyzed over 188 cases (376 pairs of mammographic images). 66 of these cases contained malinou $\equiv$ ifications. 1. table shows the results of the matching in a case level. Thus the $13.1 \%$ increase in specificity means that the algorithm cleared all the FP hits on the four images in those cases. The reasons for the loss of positive markers is that no matching pair is found because: (i) matching stripe is wrong, see variance in Fig. 3., (ii) the lack of detected microcalcification cluster or mass in one of the views, and too small credibility for the detected one (iii) the lack of microcalcification cluster in one of the corresponding views (even radiologist could not find it).

Table 1. Table of results:

\begin{tabular}{|l|c|c|c|}
\hline & $\begin{array}{c}\text { Original microcalcification } \\
\text { algorithm }\end{array}$ & $\begin{array}{c}\text { Calcifications after } \\
\text { matching reassessment }\end{array}$ & $\begin{array}{c}\text { Percentage } \\
\text { change }\end{array}$ \\
\hline \hline Sensitivity & $95.5 \%$ & $92.4 \%$ & $-3.1 \%$ \\
\hline Specificity & $0.8 \%$ & $13.9 \%$ & $+13.1 \%$ \\
\hline FP/image & $3.25 \mathrm{FP} /$ image & $1.57 \mathrm{FP} /$ image & \\
\hline
\end{tabular}

\section{Credibility calculation with MLP (Multilayered Perceptron)}

Currently a new method for determining the $P_{\text {calc }}$ value is researhed. As it can be seen on Fig. 6. a calcification cluster can be described not only by the number of calcifications and intensity parameters but by area parameters (like the area of 
microcalcifications), by shape parameters (like the average length of the major axis of the calcifications ...), distance parameters (like the average distance between the calcifications) ...

We have acquired a 1390 true positive clusters determined by the microcalcification algorithm. These clusters are considered to be true positives as they overlap a mark of radiologists. A false positive sample set was gathered from the clusters found that did not overlap radiologists' mark. 35 parameters were determined to each cluster, out of which 13 were used as an input vector to the network. 834-834 (TP/FP) clusters were used to teach a simple MLP with Levenberg-Marquart algorithm (10 neurons in the hidden layer, -1 expected for parameters gained from a FP cluster and +1 expected for parameters gained from a TP cluster). Early stop was implemented with a testing set of 254-254 clusters. The remaining 302(TP)-284(FP) clusters were used for validation of the network.

A treshold was determined so that clusters which fall below are automatically dropped as false positive hits. $1 \mathrm{TP}$ and $102 \mathrm{FP}$ clusters were dropped by the network because of the treshold. The credibility values for the remaining clusters were determinded according to the distance they resulted from the treshold and the value 1 . Fig. 5 . shows the histogram of determined credibility values.

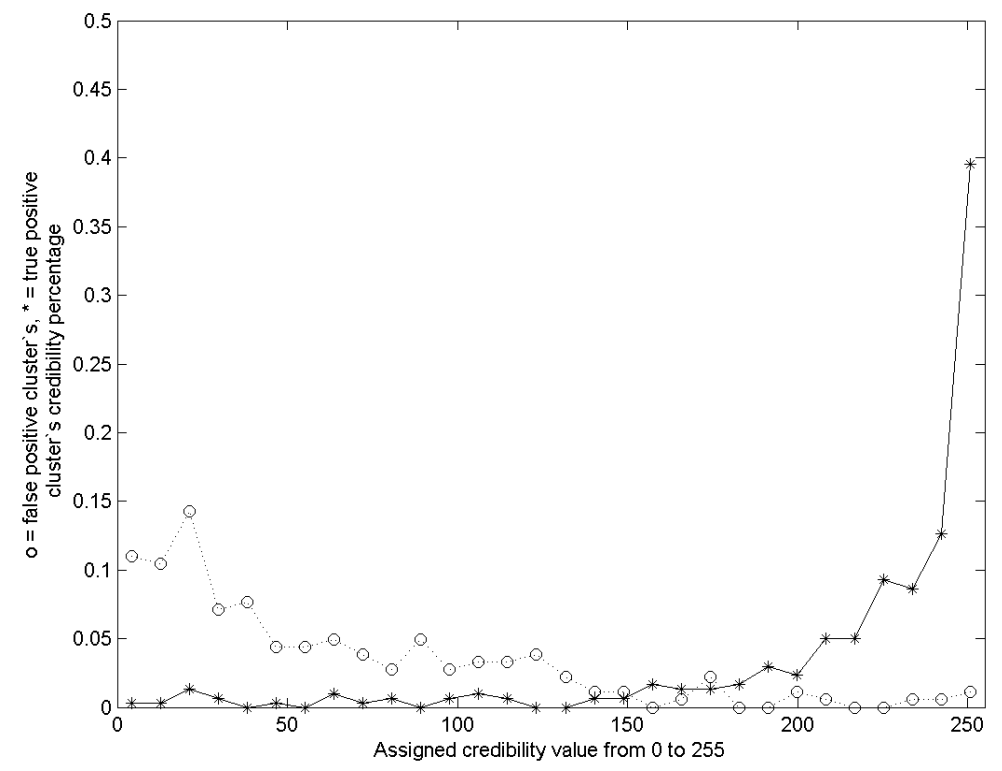

Fig. 9. The percentages of true positive and false positive cluster's credibility values 


\section{Conclusions}

The paper proposed a relatively simple way of combining the results of microcalcification detection algorithms applied for individual X-ray breast images. The joint analysis follows the procedure of skilled radiologists: if a suspicious area can be found in one view, usually its corresponding pair should be detected in the other view of the same breast. The first results - based on a a few hundred of cases - show that using this approach the number of false positive detections can be reduced significantly while the decrease of true positive hits is relatively small.

Moreover a better method to calculate the credibility was examined using microcalcification shape information, and other features like distance as well to obtain primal $P_{\text {calc }}$ value .

The proposed joint analysis system is still under testing. The improvement of the primal calcification searching algorithm, and the further analysis of the pairing (like drawing up a FROC curve) is needed. Also we intend to test the merging of the new credibility value assignment with the pairing, and see how the results can further change the $T P / F P$ ratio.

\section{References}

1. Songyang Yu, Ling Guan: A CAD System for the Automatic Detection of Clustered Microcalcifications in Digitized Mammogram Films. IEEE Trans. on Medical Imaging, Vol. 19, No. 2, February 2000.

2. B. Verma and J. Zakos: A Computer-Aided Diagnosis System for Digital Mammograms Based on Fuzzy-Neural Feature Extraction Techniques. IEEE Trans. on Information Technology in Biomedicine, vol. 5. No. 1. pp. 46-54. 2001.

3. R.J. Ferrari, R. M. Rangayyan, J. E. L. Desautels, R. A. Borges, A. F. Frre: Automatic Identification of the Pectoral Muscle in Mammograms. IEEE Trans. on Image Processing, Vol. 23, No 2, pp. 232-245, February 2004.

4. Wei-Ying Ma, B. S. Manjunath: EdgeFlow: A Technique for Boundary Detection and Image Segmentation. IEEE Trans. on Image Processing, Vol. 9, No 8, pp. 1375-1388, August 2000.

5. M. Heath, K. Bowyer, D. Kopans, R. Moore, K. Chang, S. Munishkumaran and P. Kegelmeyer: Current Status of the Digital Database for Screening Mammography. In: Digital Mammography, N. Karssemeier, M. Thijssen, J. Hendriks and L. van Erning (eds.) Proc. of the 4th International Workshop on Digital Mammography, Nijmegen, The Netherlands, 1998. Kluwer Acamdemic, pp. 457-460. 